\title{
Análisis de las decisiones estratégicas como factor clave de la innovación y competitividad de las pequeñas empresas del sector automotriz en San Luis Potosí
}

\author{
Luis Miguel Mora Guerrero ${ }^{1}$ \\ Mario Alberto Martínez Rojas ${ }^{2}$ \\ Adriana Eugenia Ramos Ávila ${ }^{3}$
}

\begin{abstract}
Resumen
Este articulo tiene como objetivo analizar y comprender las variables que afectan a las decisiones estratégicas en la pequeña empresa del sector automotriz de San Luis Potosí. El texto inicia con una introducción que contextualiza al lector sobre los antecedentes e importancia de las pequeñas empresas, posteriormente se establece el marco teórico donde se definen variables claves como son decisiones estratégicas, innovación y competitividad, cada una de ellas con sus modelos; la metodología es el siguiente punto en el desarrollo del estudio, en este ítem se plasma el método de investigación y la forma de trabajo para la realización del mismo. Los resultados y discusión es la parte del articulo donde se encuentra el desenlace de la hipótesis y de la pregunta de investigación generando análisis propios; por último, se encuentran las conclusiones donde se opina sobre el aprendizaje y los resultados del estudio, todo esto siendo de gran importancia para el sector automotriz.
\end{abstract}

Palabras clave: decisión estratégica, innovación, competitividad, sector automotriz.

\begin{abstract}
This paper aims to analyze and understand the variables that affect the strategic decisions in the small business of the automotive sector of San Luis Potosi. The text begins with an introduction that contextualizes the reader about the antecedents and importance of small companies, later establishes the theoretical framework where key variables are defined as strategic decisions, innovation and competitiveness, each of them with their models; The methodology is the next point in the development of the study, in this item the method of investigation and the form of work for the realization of the same is captured. The results and discussion is the part of the article where the outcome of the hypothesis and the research question is generated generating own analysis; Finally, we find the conclusions about learning and the results of the study, all of which are of great importance for the automotive sector.
\end{abstract}

Keywords: strategic decision, innovation, competitiveness, Automotive section.

\footnotetext{
${ }^{1}$ Licenciado en Administración de Negocios Internacionales. Profesor de la Universidad Mariana, San Juan de Pasto, Colombia. E-mail: Immg0725@hotmail.com

${ }^{2}$ Contador Público. Doctor en administración. Profesor de la Facultad de Contaduría y Administración de la Universidad Autónoma de San Luis Potosí. E-mail: amtz75@gmail.com

${ }^{3}$ Doctora en Administración. Profesor de la Facultad de Contaduría y Administración de la Universidad Autónoma de San Luis Potosí. Email: ady_ramosa@yahoo.com.mx
} 


\section{INTRODUCCIÓN}

La presente investigación se enfoca en analizar y comprender la influencia de una decisión estratégica en la competitividad y las prácticas de innovación de las pequeñas empresas del sector automotriz en San Luis Potosí; la decisión estratégica se la puede definir según Rivas (2013, p. 110) como la selección de la mejor opción para la ejecución de una actividad, dicha opción va a implicar un alto compromiso en la utilización de los recursos de una empresa u organización; las decisiones estratégicas como herramienta gerencial van a ser influyentes en la innovación que según la Organización para la Cooperación y Desarrollo Económicos (OCDE) y Oficina de Estadísticas de las Comunidades Europeas (EUROSTAT) (2005, p. 56) lo definen como llevar a cabo una idea que cree o modifique bienes o servicios, sin embargo, estos autores afirman que la innovación también puede ser aplicada en procesos administrativos y productivos. La sinergia del efecto que causa una buena decisión estratégica sobre el proceso de innovación va a generar competitividad en la organización, que según Saavedra y Tapia (2012, p. 6) lo especifican como la sumatoria de factores como la productividad, las finanzas, las relaciones publicas entre otros que llevan a la empresa a mantenerse en el mercado y generar unas ganancias.

La investigación de estos factores fundamentales de una empresa se realizó por el interés de conocer a fondo el por qué las pequeñas empresas no pueden pasar a ser grandes empresas y de igual manera saber que actividades a nivel gerencial llevan a que estas se mantengan en el mercado globalizado que es acaparado por empresas de gran envergadura con un musculo financiero muy grande y que fácilmente pueden absorberlas. La importancia e interés de estudio de estos factores se basó en conocer y explicar el contexto en el que desempeñan las pequeñas empresas, para aportar datos verídicos y actuales al sector.

A través de una investigación documental, se puede identificar y analizar la problemática central, que consiste en la carencia de decisiones estratégicas que afectan a la innovación y competitividad y por ende crecimiento en una empresa.
Las pequeñas empresas del sector automotriz de San Luis Potosí que no aplican estos factores están en riesgo de fracasar en los mercados ya que compiten con grandes empresas que si cumplen y ejecutan planes de trabajo que les asegure una estabilidad y crecimiento sostenible. Para ello es importante preguntarse ¿cuáles son los elementos, características y condiciones que las pequeñas empresas del sector automotriz de la ciudad de San Luis Potosí deben tener en cuenta para la toma de decisiones estratégicas que les permita innovar y ser competitivos en su ámbito?

Las pequeñas empresas son la base de una economía emergente, como lo es México, por lo tanto, he aquí la importancia de realizar un estudio de este tipo, para apoyar las pequeñas empresas e incrementar su potencial en el entorno que las rodea y que de este modo los empresarios y/o lectores de este articulo puedan conocer dichos factores para posteriormente realizar planes de mejora y así poder tener un crecimiento en la organización. San Luis Potosí es una ciudad industrializada, y ella alberga numerosas empresas dedicadas a la fabricación o ensamble de autopartes, por ende, esta investigación es de gran ayuda para que las pequeñas empresas locales no desaparezcan en este mercado de grandes proporciones.

\section{MARCO TEORICO}

\section{Decisión Estratégica:}

Para poder entender el concepto de decisión estratégica se debe comprender cada una de las palabras que lo componen; decisión es "Determinación, resolución que se toma o se da en una c osa dudosa” Real Academia Española (RAE) (s.a, s.p). Según Peñaloza (2010, p.228), afirma que la decisión o tomar una decisión es la evaluación entre varias alternativas para posteriormente escoger una de ellas esperando sea la correcta, dicha selección se realiza teniendo en cuenta la información que se presente en la situación; de igual manera expresa que existen tres tipos de decisiones, las que se toma en condiciones de certeza, en condiciones de riesgo y en condiciones de incertidumbre. 
La palabra estrategia, conforme a la Real Academia Española (RAE) (s.a, s.p) la define como "En un proceso regulable, conjunto de las reglas que aseguran una decisión óptima en cada momento.” Asimismo, Mintzberg (1987, p.11) opina que la estrategia tiene dos características principales, una de ellas es que la estrategia se realiza antes de que suceda el hecho o la actividad a desarrollar y la segunda característica consiste en que las estrategias se las debe plantear de manera consciente y con un propósito u objetivo definido.

Una vez definido cada una de las palabras que componen el término "decisión estratégica”, se puede decir que este factor se define como "aquellas decisiones no rutinarias que involucran gran compromiso de recursos y cuyas acciones tienen impacto en el desempeño a largo plazo de la organización.”; de igual manera también señala que "Considerar que solamente la alta dirección está capacitada para tomar decisiones estratégicas es negar lo que ellos mismos consideran fundamental: el conocimiento producido en la organización, ese que les ha permitido hoy, ser líderes de mercado.” (Rivas, 2013, p.124).

\section{Innovación:}

La innovación es un término y un factor que se debe utilizar muy a menudo en las empresas que quieran pertenecer a un mercado globalizado, según la RAE (s.a, s.p) la palabra innovación hace referencia a “ creación y modificación de un producto y su introducción en el mercado"; sin embargo, esta definición es incompleta según otros autores como es el caso de Fajardo (2015, p. 532), donde afirma que la innovación no solamente se realiza sobre un producto, sino también puede ser aplicada para modificar un problema o situación generando un beneficio económico sobre el mercado que este incursionando dicha persona u organización.

Se define a la innovación tecnológica como la sinergia y aplicación de las actividades derivadas de etapas científicas, comerciales, organizacionales, financieras entre otras, incluyendo la adquisición de nuevos conocimientos; dichas actividades serán aplicadas sobre productos o procesos que los van a llevar a ser mejores o incluso crear unos nuevos OCDE (2002 p.17).
Una vez se esté aplicando la innovación, en el transcurso del proceso pueden aparecer otras actividades innovadoras aplicables al producto o procesos, tales como "know-how" e implementación de nuevas herramientas tecnológicas OCDE y EUROSTAT (2005, p.23).

Según el Centro Europeo de Empresas e Innovación de Ciudad Real (CEEI) (2007, p.6) en su publicación denominada "Manual de Innovación”, hace referencia de la innovación como la generación de beneficios económicos en empresas u organizaciones a través de llevar a cabo una idea que cree productos, servicios y procesos nuevos, o mejorados para la incursión en un mercado comercial.

La OCDE Y EUROSTAT (2005, p. 56) definen en el Manual de Oslo a la innovación como la mejora o producción inédita de un bien, servicio, proceso, método de comercialización, o de un método organizativo dentro de una empresa u organización para sobresalir en el medio o mercado en el cual se desempeñe.

Los autores clásicos tienen otra visión para hablar de innovación; para Schumpeter (1934), la innovación es un factor que cambia la economía y dicho factor se divide en innovaciones radicales e innovaciones incrementales; la primera genera grandes y significativos cambios en el mundo, mientras que la segunda división completa el proceso del cambio, y se centran principalmente en la industria; debido a que la innovación se divide en cinco ítems, se los nombran y conceptualizan a continuación (ver Figura 1). 
Figura 1. Tipos de innovación.

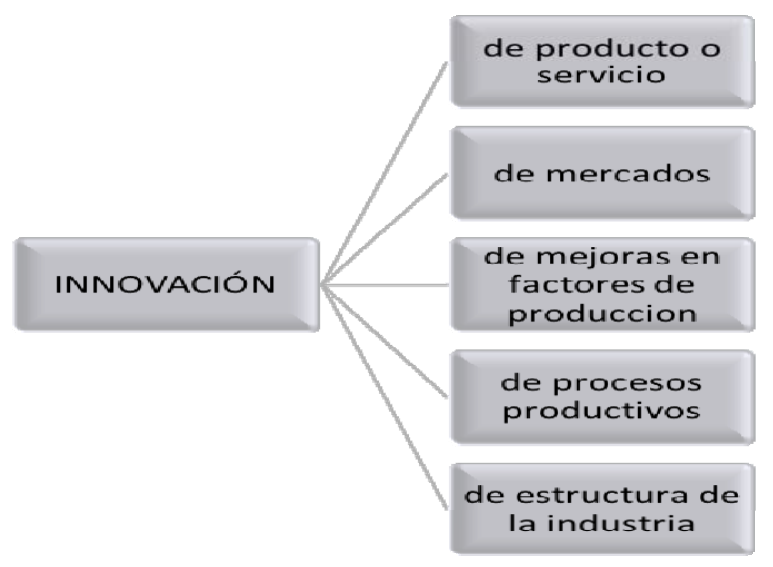

Fuente: Elaboración propia. Adaptado de: Schumpeter, J. (1934), The Theory of Economic Development.

Figura 2. Tipos de innovación en la actualidad.

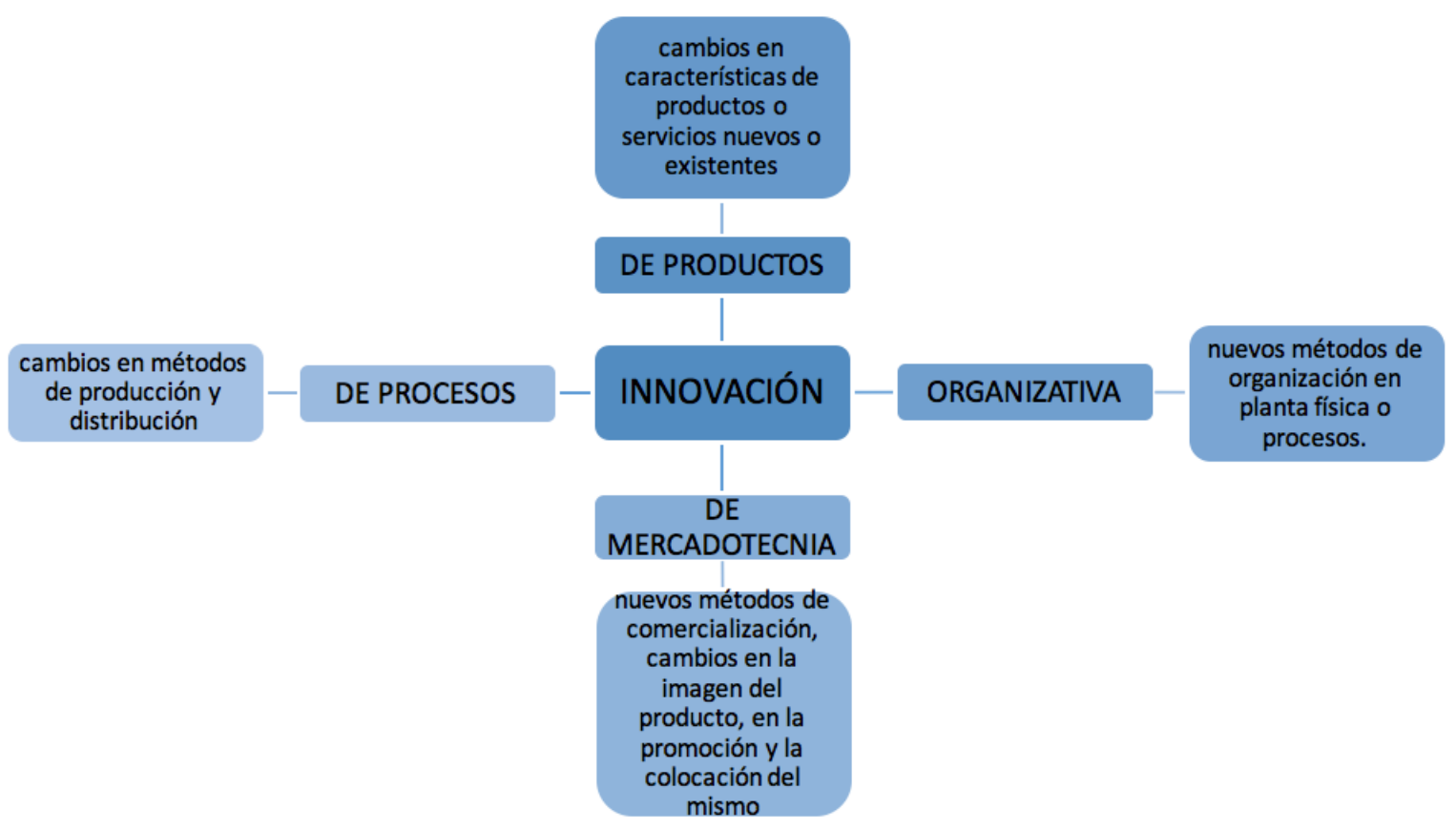

Fuente: Elaboración propia. Adaptado de: Manual de Oslo, OCDE Y EUROSTAT. (2005, p.23).

La innovación de producto o servicio se enfoca principalmente en la creación o búsqueda de una mejora para un producto o servicio y de este modo superar las expectativas del mercado; la innovación de mercados se define como el cambio físico o de presentación para el producto o servicio, de este modo hacerlo más llamativo para los clientes, consumidores o usuarios; la innovación de mejoras en factores de producción trata sobre buscar la manera para que el bien o el servicio pueda realizarse a un costo menor, con menor material o simplemente buscar la forma de que la empresa incremente su efectividad en la producción; la innovación de procesos productivos se refiere a encontrar el método para que la empresa optimice recursos de toda clase a través de nuevos procesos o a través de mejorar los ya existentes; la innovación de 
estructura de la industria se enfoca en implementar nuevos sistemas productivos para mejorar la producción y cumplir con las necesidades de los clientes, por ejemplo los clusters.

Así mismo anudando en lo anterior, la OCDE Y EUROSTAT (2005, p. 23) subdividen a la innovación en innovaciones de producto, innovaciones de proceso, innovaciones organizativas e innovaciones de mercadotecnia (ver Figura 2).

\section{Competitividad:}

El termino competitividad es uno de los elementos que permiten a la empresa tener un crecimiento, de este modo existen muchos autores que hablan de este tema y cada uno de ellos tiene una percepción distinta sobre el significado del término competitividad. Uno de los significados de este concepto es que "La competitividad es un concepto relativo, muestra la posición comparativa de las empresas al utilizar la misma medida de referencia.” (Pérez, 2008, citado por Mosquera 2010, p. 29)

Nedić, Lepojević, Despotović, y Cvetanović. (2016) consideran que el concepto de competitividad está directamente relacionado a la diferencia positiva que generan las personas, empresas, países y regiones frente a otros similares; dicha diferencia hará a cada uno de ellos más innovadores y más importantes en el mercado en el que se desenvuelvan fortaleciendo su propuesta de valor en el servicio o producto que ofrecen.

“(...) La competitividad es la capacidad de las empresas para competir, crecer y ser rentable (...)" (Martin, (s.a), p. 2-1). De la misma manera, la competitividad es la consecución de unos objetivos financieros y económicos que tienen las empresas, individuos o regiones para sobresalir en un mercado, siendo sostenibles y siendo mejores que su competencia produciendo mayor volumen utilizando menos recursos. (Clipa e Ifrim, 2016).

Saavedra y Tapia (2012, p. 6) señalan que "La competitividad de una empresa depende de la productividad, la rentabilidad, la posición competitiva, la participación en el mercado interno y externo, las relaciones inter-empresariales, el sector y la infraestructura regional.”

Como se expresó en el inicio de este artículo, el concepto de competitividad es muy amplio y tiene diversas connotaciones dependiendo del autor que aborde el tema sin embargo los autores citados para definir este concepto coinciden con que la competitividad involucra unos factores como es la rentabilidad, sostenibilidad, competencia y mercados, donde aportan que para que una empresa o una nación sea competitiva necesita ser rentable y tener una sostenibilidad continua sobresaliendo en el mercado que se desempeñen; sin embargo, algunos de ellos profundizan $\mathrm{y}$ adicionan factores tales como la productividad, la innovación, la tecnología, la macroeconomía y la microeconomía como variables influyentes en la competitividad. (Nedić et al, 2016; Mosquera, 2010; Martin, s.a; Clipa e Ifrim, 2016; Saavedra y Tapia, 2012).

Por otra parte, el Banco Interamericano de Desarrollo (BID) (2001) observa que, la competitividad ha sufrido unos cambios significativos, sobre todo en la región latinoamericana donde ha pasado de un concepto mercantilista a un concepto más holístico que incluyen factores macroeconómicos, financieros, de talento humano e innovación que juntos representan el progreso y el incremento de la productividad de una empresa, industria o de un país. Este concepto se adapta a la afirmación que realiza Porter (1991) donde afirma que para que un país sea más competitivo, la demanda interna de dicho país debe ser exigente, exclusiva y sofisticada; de este modo, las empresas productoras de los bienes van a ser más competitivas para satisfacer las necesidades de dicha demanda teniendo más posibilidad de internacionalizarse; esta afirmación incluye todos los factores mencionados por el BID.

Saavedra y Tapia (2012) afirman que dentro de las pequeñas empresas (PYME'S) existe un enfoque sistémico de la competitividad y esta a su vez está dividida en cuatro vectores que giran alrededor de la competitividad, así como se observa en la Figura 3: 
Figura 3. Factores claves de la competitividad con enfoque sistémico.

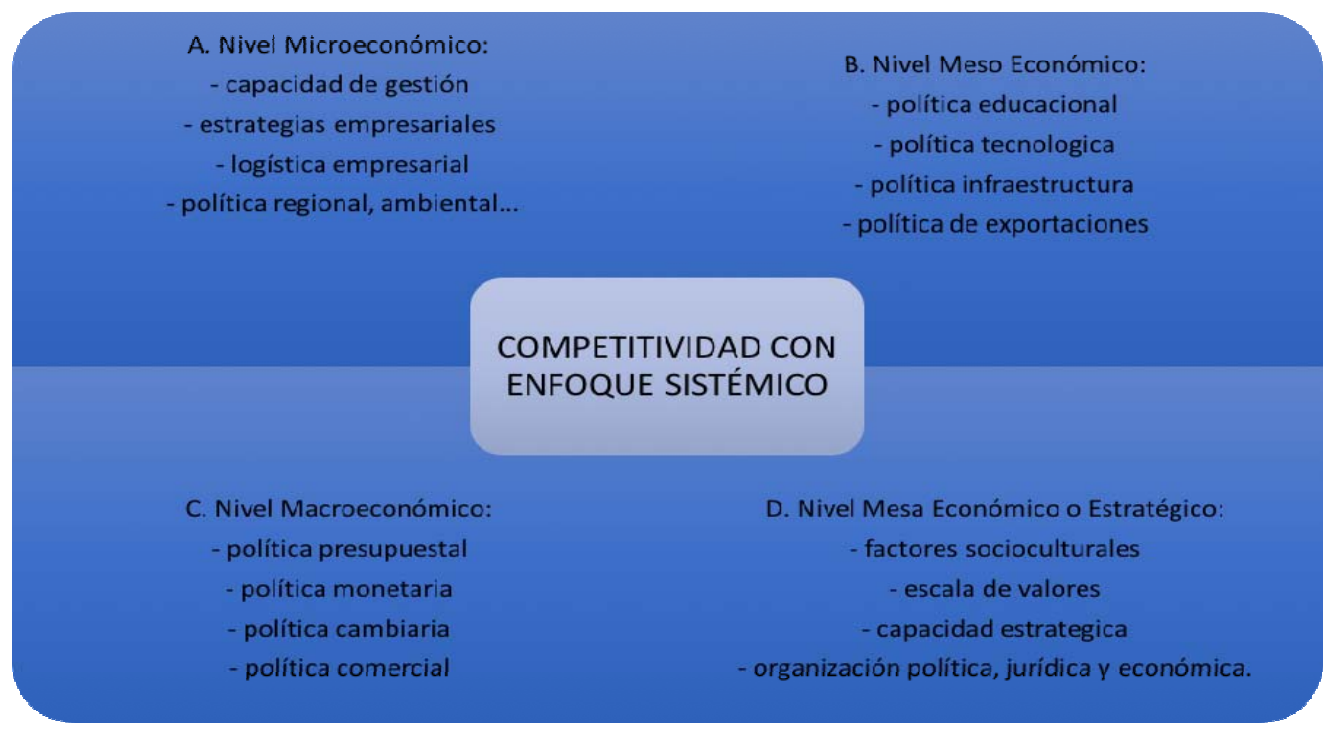

Fuente: Elaboración propia. Adaptado de: Saavedra y Tapia, 2012.

a. Nivel microeconómico: consta de todas aquellas variables que son decisiones internas de la empresa y pueden ser modificadas y constituidas por la decisión estratégica.

b. Nivel meso económico: son los factores que miden la relación entre los agentes económicos y la macroeconomía.

c. Nivel macroeconómico: son los factores relacionados con la economía global o mundial, factores externos de las empresas que ellas no pueden modificar ni alterar, dentro de estas variables están la política presupuestal, política monetaria, política cambiaria, política comercial entre otras.

d. Nivel meta económico o estratégico: es el nivel que aporta factores sociales a la economía.

Una manera de generar competitividad a las empresas y en general a las pequeñas empresas o PYMES según Delgado, Mercedes, Porter, y Scott (2010) afirman que los cluster son un método para el surgimiento de dichas empresas; estos autores definen a los clusters como un grupo o aglomeración de empresas ubicadas en un sector geográfico contiguo que se apoyan para sus procesos productivos generando sinergia a través de la cercanía de su ubicación.
Según (Porter, 2015) plantea que para que una empresa consiga la competitividad, se debe enfocar principalmente en tres estrategias que son:

1.- Liderazgo global en costos: esta estrategia, según el autor, permite que la empresa pueda evitar las amenazas del mercado ya que se enfoca en controlar fuertemente los costos variables y costos fijos en la producción y funcionamiento organizacional, sin descuidar la calidad de los servicios o productos. La consecución de los costos bajos en la compañía va a proporcionar a la empresa que tenga dominio y control sobre las cinco fuerzas que Porter (2009, p. 31) establece en su libro "ser competitivo" los cuales son: rivalidad entre competidores existentes, amenaza de nuevos aspirantes, poder de negociación de los proveedores, poder de negociación de los compradores y amenaza de productos o servicios sustitutos.

2.- Diferenciación: esta estrategia sirve para darle el valor agregado a un servicio o al producto, dicha diferencia los clientes o usuarios lo van a percibir como únicos y por lo tanto va a ser una ventaja que va a tener la compañía frente a sus competidores en el mercado. Una vez la empresa consiga esta característica de diferencia, va a lograr una fidelidad de sus clientes donde se reduce notablemente la sensibilidad del precio 
esta estrategia permite que se cree una barrera de entrada para los competidores directos y sustitutos motivo por el cual los clientes son fieles a la marca y al producto o servicio.

3.- Enfoque o concentración: esta estrategia es aplicable para un grupo de clientes o target especifico con características especiales, busca satisfacer sus necesidades con un servicio y un producto de excelencia, pero con el esfuerzo de ofrecerlo a un precio bajo. Esta estrategia aplica el liderazgo en costos y por lo tanto puede obtener rendimientos más altos que los del promedio de la industria.

\section{MARCO CONTEXTUAL}

El presente proyecto se lleva a cabo en la ciudad de San Luis Potosí, actual capital del estado de San Luis Potosí. La población total de la ciudad estimada en 2010 según datos del Instituto Municipal de Planeación del Ayuntamiento de San Luis Potosí (2011, p.4) es de 772,604 habitantes con una tasa de crecimiento poblacional de 1.94 entre los años de 1990 y 2010, sus generalidades se ilustran en la Tabla 1 y su ubicación geográfica en la Figura 4.

Tabla 1. Generalidades de San Luis Potosí.

\begin{tabular}{|c|c|}
\hline País & México \\
\hline Estado & San Luis Potosí \\
\hline Región & Centro \\
\hline Ubicación & $100^{\circ} 50^{\prime \prime}$ Longitud Oeste y $22^{\circ} 09^{\prime \prime}$ de Latitud Norte \\
\hline Temperatura & $16.8^{\circ}$ (temperatura media anual) \\
\hline Altitud & $1.860 \mathrm{msnm}$ \\
\hline Superficie & $1.471 .7 \mathrm{~km}^{2}$ \\
\hline Fundación & 1.592 \\
\hline
\end{tabular}

Fuente: Elaboración propia. Adaptado de: Enciclopedia de los municipios y delegaciones de México, Estado de San Luis Potosí. 
Figura 4. Ubicación geográfica.

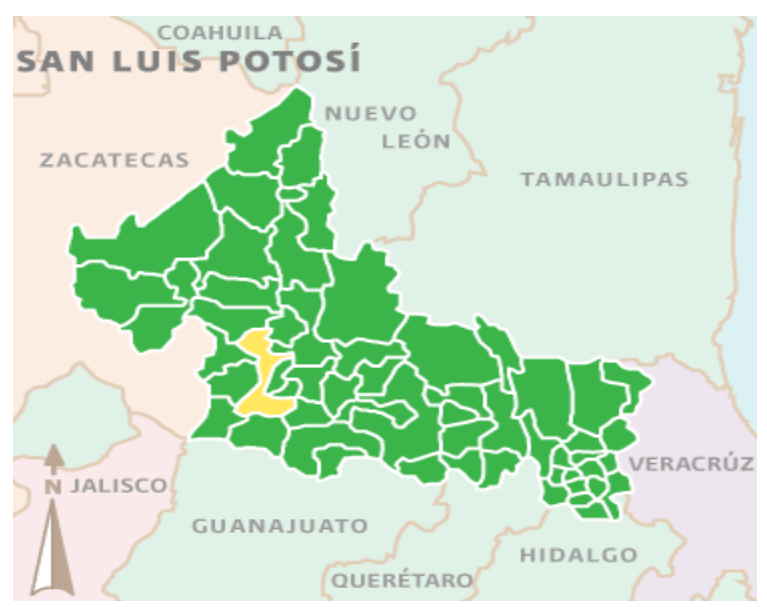

Fuente: Enciclopedia de los municipios y delegaciones de México, Estado de San Luis Potosí.

\section{Sector automotriz:}

Según la Secretaria de Desarrollo Económico de San Luis Potosí en su publicación Industria Automotriz y de Autopartes del Estado de San Luis Potosí (2012, p. 1155), afirman que el sector automotriz es uno de los sectores manufactureros más dinámicos económicamente hablando comparado con otros sectores de ramas industriales; en México, la producción de automotores livianos significó el 3.3\% de la producción mundial para el año 2011 y de esta cifra más del $80 \%$ se destinaron para la exportación a mercados extranjeros. Para este mismo año, esta industria empleó en todo el país a 1.7 millones de personas que corresponde al $45 \%$ de los trabajadores registrados en el instituto mexicano de seguridad social. El sector automotriz en México es uno de los que más aporta al país en lo que corresponde a exportaciones puesto que abarca un $22.5 \%$ de las exportaciones totales ubicándose por encima de las exportaciones de los productos derivados del petróleo manteniendo un superávit comercial; de igual manera, se destaca la industria de autopartes que es una de las más diversificadas del mundo, dicha industria exporta el $60 \%$ del total de su producción y de dicho valor el $89 \%$ se destina al mercado estadounidense lo que deja ver que Estados Unidos es el principal cliente de esta industria.
El desarrollo que se puede observar en el sector automotriz y en la industria manufacturera de autopartes ha sido gracias a los convenios comerciales que tiene México con el resto del mundo siendo una oportunidad para todas las empresas, pero sobre todo para las pequeñas empresas de este sector; se puede hablar de convenios con alrededor de 43 países entre los que se encuentra el tratado de libre comercio con Estados Unidos y Canadá que son los principales clientes de la industria.

A nivel local, desde la década de los años cincuenta han venido asentándose en el estado empresas de gran envergadura que han generado empleo y han realizado una gran inversión, al llegar empresas fabricantes de autopartes y ensambladoras se han generado clusters que fortalece el sector, por lo tanto, el estado de San Luis Potosí ha sido una ubicación estratégica para el desarrollo de la industria automotriz. Para el año 2008, la ciudad aporto el 3.2\% del producto total nacional de la industria automotriz ubicándose en la casilla once después de Baja California (ver Grafica 1); la producción para este año solamente fue realizada por ochenta y tres (83) empresas, sin embargo, para el año 2012 creció el número de empresas a ciento tres (103) de las cuales el $19 \%$ corresponden a pequeñas empresas. Muchas de estas empresas están vinculadas o tienen convenios con grandes compañías ensambladoras o productoras de autopartes. 
Grafica 1. Producción Buta Total por estados de la industria automotriz.

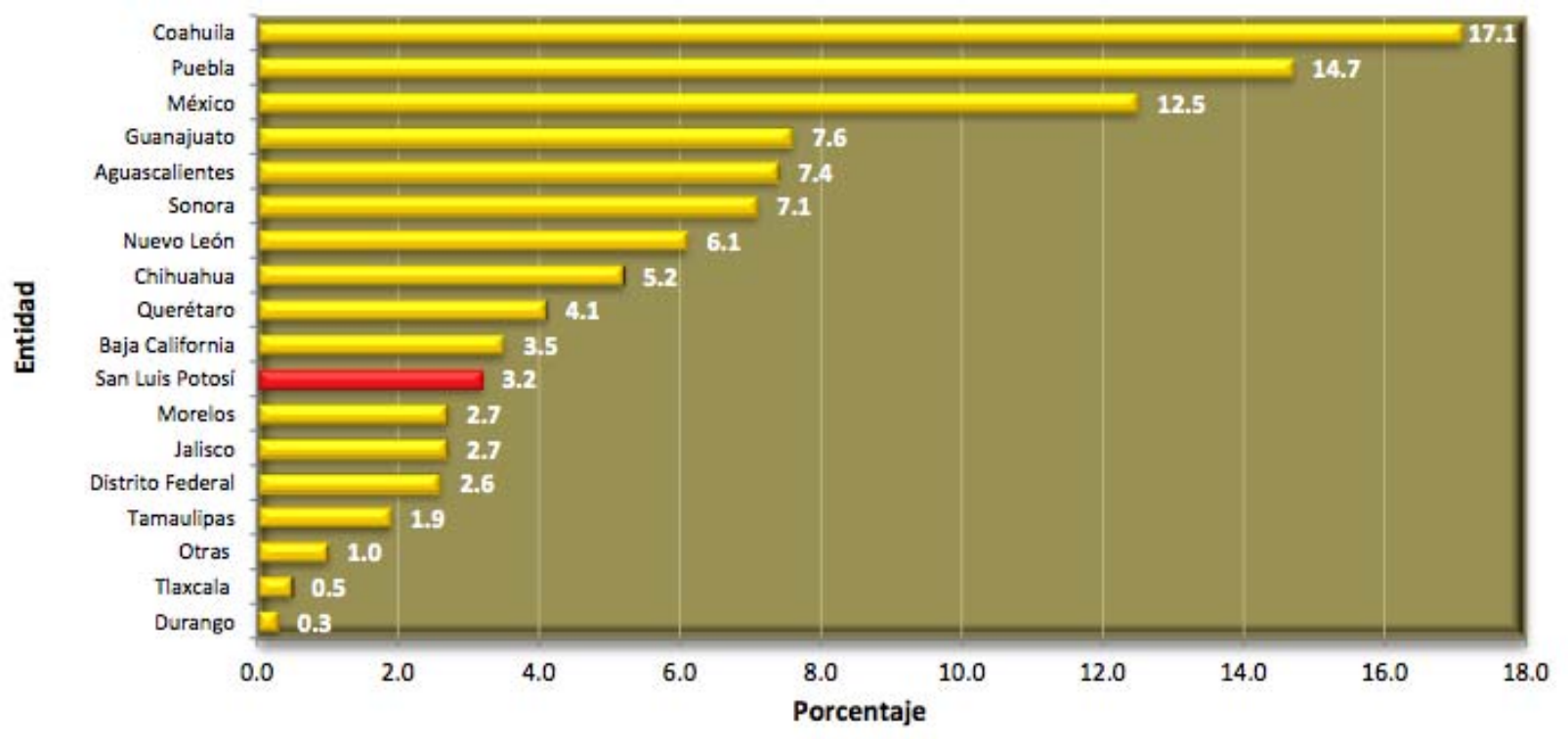

Fuente: Censos Economicos, INEGI. 2009. Citado por: Secretaria de Desarrollo Economico. 2015. P. 44.

En la ciudad de San Luis Potosí se sitúan el 69\% de las empresas de la industria automotriz y de autopartes del estado. Del total de la producción bruta en la ciudad, las empresas de fabricación de autopartes aportaron el 71.65\%, estas empresas pertenecen al tipo 2 y 3 de clasificación y son en su mayoría de inversión nacional a diferencia de las empresas tipo 1 que en su mayoría son de inversión extranjera. Las empresas tipo 1 (T1) son aquellas que proveen de autopartes a las grandes ensambladoras del país, el tipo 2 (T2) son dedicadas a fabricar refacciones especializadas para las empresas T1 y las empresas tipo 3 son aquellas que se dedican a producir artículos de menor complejidad para las empresas T2.

En el país mexicano hay establecidas al año 2013 cuatro millones doscientos treinta mil setecientos cuarenta y tres (4.230.743) empresas de todos los sectores, las MIPYMES del sector manufacturero ocupan el $11.6 \%$ del total anteriormente mencionado, estas empresas ocupan el $11.6 \%$ del total del personal ocupado en México. Las pequeñas empresas que son el objeto de estudio, dentro de la industria manufacturera al año 2013 se calculan veinte mil cuatrocientos cincuenta y cinco (20.455). en San Luis Potosí se calculan en el mismo año la existencia de ocho mil ochocientos cincuenta y dos (852) empresas que representan el $10 \%$ del total de compañías existentes, ocupando a ciento veinticinco mil once personas (125.011). En San Luis Potosí predominan las micro empresas con un $96.1 \%$ del total, seguido por las pequeñas empresas con un $3 \%$ y por ultimo las medianas y grandes empresas con un $0.7 \%$ y $0.3 \%$ respectivamente.

\section{METODOLOGIA}

Para la construcción del presente artículo se adoptó un enfoque cualitativo y es una investigación de tipo documental; se consultó en bases de datos como EBSCO, REDALYC, SCIELO donde se encontró artículos científicos, en la base de datos EBRARY se encontraron libros virtuales y también se consultó páginas de organismos oficiales como la Organización para la Cooperación y Desarrollo Económicos (OCDE), Oficina de Estadísticas de las Comunidades Europeas (EUROSTAT) y la Real Academia Española (RAE) que ayudaron al desarrollo del marco teórico como también para plasmar el análisis de los resultados. 
Según Hernández, Fernández y Baptista (2010, p.7) afirman que el enfoque cualitativo de una investigación no utiliza datos numéricos para resolver las preguntas de investigación, sino que hace uso de la literatura para entender y resolver dichas preguntas; en esta clase de estudios, la hipótesis y las preguntas de investigación pueden desarrollarse antes, durante o después de haber recolectado y analizado los datos.

\section{RESULTADOS Y DISCUSIÓN}

A continuación, se presenta en la Figura 5 los resultados de la investigación documental, en donde se resume en un diagrama las siguientes variables: decisión estratégica, innovación y competitividad, además de las relaciones que existen entre ellas.

Figura 5. Resultados de investigación.

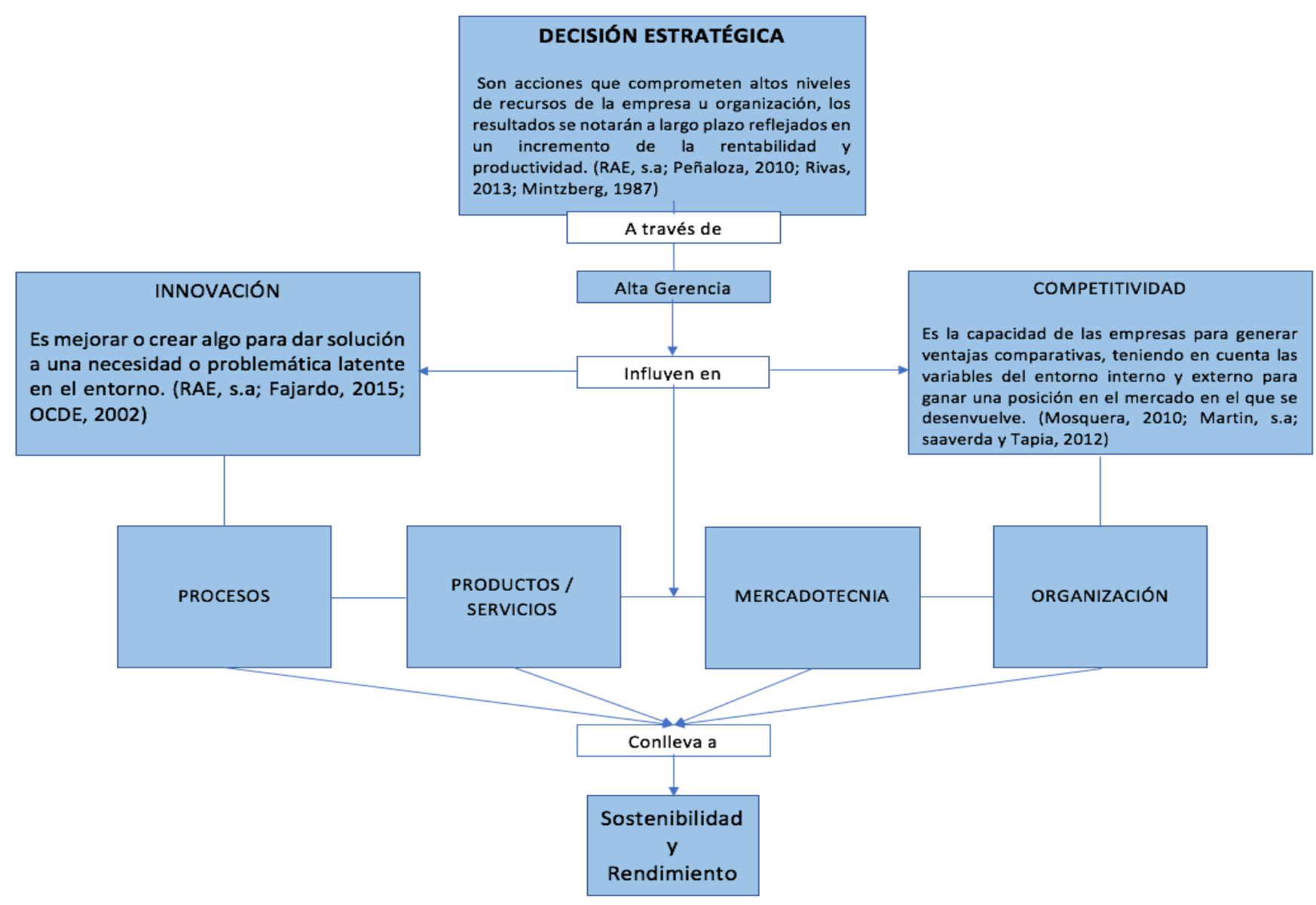

Fuente: Elaboración propia. 
Como resultado de la investigación, se puede decir que las decisiones estratégicas son indispensables en el desarrollo de las actividades de una empresa, puesto que a través de ellas se toman decisiones que pueden afectar directamente el futuro de la compañía debido a que los efectos de las decisiones estratégicas se evidencian a largo plazo (cinco años o más). En el presente estudio, la influencia de las decisiones estratégicas en la innovación y competitividad de las pequeñas empresas del sector automotriz de San Luis Potosí es alta, ya que estas empresas utilizan a diario herramientas tecnológicas con las que desarrollan sus actividades principales; a través de las decisiones estratégicas los empresarios decidirán qué tan innovadores son o dejan de ser, una vez se aplique la innovación, la empresa pasa a ser competitiva y a ocupar un puesto en el mercado de autopartes de la ciudad que es muy competitivo por el nivel de inversión que manejan los competidores.

Los encargados de tomar las decisiones estratégicas en las empresas son los gerentes o la alta gerencia, por lo tanto las decisiones se aplicarán de forma global en la compañía, es decir, se ejecutarán en todas las áreas de la empresa, por ende se puede decir que existirá una sinergia o conexión en todos los procesos ya sean administrativos, comerciales o productivos; sin embargo, las decisiones deben ir de la mano del estilo de liderazgo que adopten los directivos, se sugiere tener una retroalimentación entre colaboradores y directivos para conocer realmente los efectos y alcances de las estrategias adoptadas. Al momento que ya se hayan aplicado las decisiones estratégicas, se puede evaluar los resultados generados en la empresa y se podría decir si hubo o no el crecimiento sostenible, el aumento del rendimiento y la rentabilidad.

\section{CONCLUSIONES}

En la actualidad, el secreto para que las empresas puedan mantenerse en el mercado es tener una visión hacia el futuro para así poderse adaptar a los cambios y exigencias que día a día se presentan en el mercado en el que se desempeñan, a eso se le podría llamar innovación, estar un paso más delante de los competidores y de las expectativas que tienen los clientes.
La correcta aplicación de las decisiones estratégicas lleva a algo que se denomina mejora continua y calidad total, estos factores combinados forman a una empresa competitiva y capaz de sortear los desafíos del mercado global.

Se puede decir que la competitividad es la finalidad de toda empresa, se puede lograr a través de ejecutar correctamente factores humanos, técnicos y de procesos que permitan a la empresa posicionarse, tener un buen lugar en el mercado y de igual manera ser mejores que sus competidores. Las pequeñas empresas del sector automotriz en San Luis Potosí deben aprovechar las oportunidades del entorno, como los cluster's, donde pueden potencializar sus fortalezas y por qué no pasar a ser grandes empresas competitivas y que aporten a la economía local, regional y nacional.

\section{Referencias}

Ayuntamiento de San Luis Potosí. (s.a). Enciclopedia de los municipios y delegaciones de México: Estado de San Luis Potosí. Consultado en línea a través de http://siglo.inafed.gob.mx/enciclopedia/EMM24sanluis potosi/municipios/24028a.html

Banco Interamericano de Desarrollo (BID) (2001). Competitividad: el motor del desarrollo informe 2001: progreso económico y social en América latina. Washington D.C, Estados Unidos: IDB bookstore.

Centro Europeo de empresas e Innovación de Ciudad Real (CEEI). (2007). Manual de Innovación: Guía Práctica de gestión de la I+D+I para Pymes. Recuperado el 10/07/17 a través de http://www.innovacion.cl/wpcontent/uploads/2013/10/manual_de_innovacion_para_ pymes.pdf.

Clipa, R. y Ifrim, M. (2016). measuring regional competitiveness. the case of Romania. Annals of The University of Oradea, Economic Science Series, 25(1), 103-111. 
Delgado, Mercedes, Porter, y Scott. "Clusters and Entrepreneurship.” Journal of Economic Geography 10.4 (2010): 495 -518. Recuperado Web. Jun 212017. https://academic.oup.com/joeg/article-

abstract/10/4/495/913653/Clusters-andentrepreneurship

Estado de San Luis Potosí, Secretaria de Desarrollo Económico. (2012). Industria automotriz y de autopartes del estado de San Luis Potosí.

Estado de San Luis Potosí, Secretaria de Desarrollo Económico. (2015). Las micro, pequeñas y medianas empresas en México y San Luis Potosí.

Fajardo, G; H, M R; (2015). Innovación. Revista Médica del Instituto Mexicano del Seguro Social, 53() 532$533 . \quad$ Recuperado de http://www.redalyc.org/articulo.oa?id=457744939001. Junio 262017

Hernández, R., Fernández, C., Baptista, P. (2010). Metodología de la investigación. México D.F. Mcgraw-hill / interamericana editores, s.A. de C.V.

Instituto Municipal de Planeación, Ayuntamiento de San Luis Potosí. (2011). Municipio de San Luis Potosí en Cifras. Consultado en línea a través de http://www.implansanluis.gob.mx/descargas/slp.cifras. pdf. 6/07/17.

Martin, R. (2003). A study on the factors of regional competitiveness. Recuperado de Internet 10/07/17. http://ec. europa. eu/regional_policy/sources/docgener/studies/pdf.

Mintzberg, H. (1987). The strategy concept I: Five ps for strategy. California Management Review. Recuperado de

http://journals.sagepub.com/doi/abs/10.2307/41165263 . 26/06/17

Mosquera Aldana, Vlashskiv; (2010). competitividad de la pequeña empresa del sector ornamentales en atlixco, México. Cali, Colombia: Universidad Libre. Entramado, Julio-Diciembre, 26-35.
Nedić, V., Lepojević, V., Despotović, D., y Cvetanović, D. (2016). Competitiveness of the Selected Balkan Countries in the Period 2006-2015. Marketing (03543471), 47(4), 278-292.

Organización para la Cooperación y Desarrollo Económico (OCDE). (2002). Manual de Frascati: Propuesta de Norma Práctica para Encuestas de Investigación y Desarrollo Experimental. Consultado en línea a través de http://www.idi.mineco.gob.es/stfls/MICINN/Investigac ion/FICHEROS/ManuaFrascati-2002_sp.pdf. 3/07/17.

Organización para la Cooperación y Desarrollo Económico (OCDE) y Oficina de Estadísticas de las Comunidades Europeas (EUROSTAT). (2005). Manual de Oslo: Guía para la Recogida e Interpretación de Datos sobre Innovación. Consultado en línea a través de http://www.itq.edu.mx/convocatorias/manualdeoslo.pd f. 3/07/17.

Peñaloza, M; (2010). teoría de las decisiones. perspectivas, Enero-Junio, 227-240.

Porter, M. (2015). Estrategia competitiva: técnicas para el análisis de los sectores industriales y de la competencia (2a. ed.). Distrito Federal, MÉXICO: Grupo Editorial Patria. ProQuest ebrary. Web. 14 June 2017

Porter, M. (1991). La ventaja competitiva de las naciones. Buenos Aires, Argentina: Ed. Vergara.

Porter, M. (2009). Ser competitivo edición actualizada y aumentada. Barcelona, España: Ediciones Deusto.

Real Academia Española. (2017). Diccionario de la Real Academia Española. Consultado en línea a través de http://www.rae.es/. 10/07/17.

Rivas, L. (2013). Exploración sobre las decisiones estratégicas desde el pensamiento complejo. Universidad y Empresa, Julio-Diciembre, 107-129. Recuperado 03-07-2017. 
Saavedra, M., Tapia, B. (2012). El Entorno Sociocultural y la Competitividad de la PYME en México. (Spanish). Panorama Socioeconómico, 30(44), 4-24.

Schumpeter, J. (1934), The Theory of Economic

Development., Cambridge, Massachusetts. Harvard University Press 